\title{
BMJ Open Orthotics and taping in the management of vertebral fractures in people with osteoporosis: a systematic review
}

\author{
Victoria A Goodwin, ${ }^{1}$ Abigail J Hall, ${ }^{1}$ Emily Rogers, ${ }^{2}$ Alison Bethel ${ }^{1}$
}

To cite: Goodwin VA, Hall AJ, Rogers $\mathrm{E}$, et al. Orthotics and taping in the management of vertebral fractures in people with osteoporosis: a systematic review. BMJ Open 2016;6:e010657. doi:10.1136/bmjopen-2015010657

- Prepublication history and additional material is available. To view please visit the journal (http://dx.doi.org/ 10.1136/bmjopen-2015010657).

Received 24 November 2015 Accepted 10 February 2016

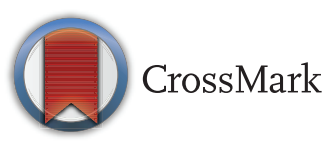

${ }^{1}$ NIHR CLAHRC South West Peninsula, University of Exeter, Exeter, UK ${ }^{2}$ Royal Devon and Exeter NHS Foundation Trust, Exeter, UK

Correspondence to Dr Victoria A Goodwin; V.goodwin@exeter.ac.uk

\section{ABSTRACT}

Objective: To establish the current evidence base for the use of orthotics and taping for people with osteoporotic vertebral fracture (OVF).

Design: Systematic review of quantitative and qualitative studies.

Data sources: Medline, Medline-In Process, EMBASE, AMED, CINAHL, PEDro, TRIP, EThOS, ProQuest Dissertations and Theses and Cochrane (CDSR, DARE, CMR, HTA, EED) plus Cochrane Central, UK Clinical Research Network portfolio, Controlled Clinical Trials register and the Australian and New Zealand Clinical Trials register.

Eligibility criteria for selecting studies: All study designs were considered if they reported in English and evaluated the impact of using an external support, such as a spinal brace, orthosis or postural tape, with adults with OVF. All outcomes were considered.

Results: Nine studies were included comprising two parallel-group randomised controlled trials, four randomised cross-over trials, two before-after (single arm) studies and a parallel group observational study. No qualitative studies were identified. A wide range of outcomes assessing impairments, activities and participation were assessed but the findings were mixed. The quality of studies was limited.

Conclusions: The current evidence for using orthotic devices or taping for people with OVF is inconsistent and of limited quality and therefore careful consideration should be taken by clinicians before prescribing them in practice.

Systematic review registration number: CRD42015020893.

\section{INTRODUCTION}

Symptomatic osteoporotic vertebral fractures (OVF) affect 1 in 6 women and 1 in 12 men during their lifetime. ${ }^{1}$ In Europe the annual incidence of OVF is 10.7 and 5.7 per thousand in women and men, respectively. ${ }^{2}$ In the USA, 700000 people are reported to have an OVF each year, ${ }^{3}$ however, these figures may underestimate the actual size of the problem as most OVF go unreported and, therefore, undiagnosed. ${ }^{4}$ With the

\section{Strengths and limitations of this study}

- The review comprised a broad search strategy, including grey literature, to maximise the capture of all of the relevant literature.

- No qualitative studies have been undertaken to establish the experiences of using people with osteoporotic vertebral fractures using orthotics or braces.

- The included studies were generally of unclear risk of bias using the Cochrane tool.

prevalence of osteoporosis predicted to increase by 2021, a rise in associated fractures is also likely. ${ }^{5}$

OVF have personal, societal and economic costs. ${ }^{6}$ Patients experience severe pain in the acute phase but also up to 2 years postfracture. ${ }^{7}$ Increased disability and depression, and a reduced quality of life are also reported. ${ }^{8}$ Up to a fifth will suffer a subsequent vertebral fracture within a year ${ }^{9}$ and there is an increased risk of mortality. ${ }^{10}$ The use of primary care services has been found to be 14 times that of the general population, ${ }^{11}$ with $8 \%$ of people with OVF requiring hospitalisation. ${ }^{12}$ In the USA, the management OVF has an annual estimated cost of $\$ 13.8$ billion. $^{1}$

Guidelines for the non-medical and nonsurgical management of OVF are conflicting. The American Academy of Orthopaedic Surgeons (AAOS) ${ }^{13}$ report inconclusive evidence in relation to rehabilitative interventions whereas the Scottish Intercollegiate Guidelines Network (SIGN) ${ }^{14}$ recommend electrical field therapy and supervised exercise programmes. There are a variety of external support devices available including rigid thoracolumbar spinal orthoses (TLSO) or hyperextension braces that are often used in the management of OVF, however, they are not recommended in current guidelines. ${ }^{13}$ Bracing is reported to assist healing when worn for up to 3 months but when 
worn for an extended period can result in muscle atrophy, postural muscle weakness ${ }^{15}$ and skin problems. ${ }^{16}$ This said, these devices are used by the people with OVF and clinicians continue to prescribe them, although practice varies.

In view of recently published studies, this review aimed to identify and update the current evidence base for the use of orthotics and taping for people with OVF.

\section{METHODS}

We used the preferred reporting items for systematic reviews and meta-analyses (PRISMA) as a guideline for reporting this study. A predefined protocol was registered with PROSPERO (CRD 42015020893).

\section{Identification and selection of studies}

We included primary studies that used quantitative or qualitative methods evaluating the impact of using an external support, such as a spinal brace, orthosis or postural tape, with adults with OVF. We were interested in outcomes relating to the WHO International Classification of Functioning, Disability and Health (ICF) domains of body structure and function, activities and participation. We were also interested in the experiences and perceptions of users of the external support.

We excluded studies that involved traumatic vertebral fractures, non-vertebral fractures and those involving children, reviews and opinion papers, studies published only as an abstract and those where full text was not available in English. We also excluded controlled studies where the intervention also included surgical, pharmacological and rehabilitation interventions, except where these were provided to intervention and comparator participants. For non-controlled studies, only those where the evaluation related to the orthotic device/tape were included.

The search strategy (see online supplementary file) was applied from 1980 to April 2015 to Medline, Medline-In Process, EMBASE, AMED, CINAHL, PEDro, TRIP, EThOS, ProQuest Dissertations and Theses and Cochrane (CDSR, DARE, CMR, HTA, EED). In addition we searched clinical trials databases, including Cochrane Central, UK Clinical Research Network portfolio, Controlled Clinical Trials register and the Australian and New Zealand Clinical Trials register. We also undertook forward and backward citation checking.

The search and screening process was managed using Endnote. Two reviewers independently screened titles and abstracts. Full-text papers were then screened against the eligibility criteria independently by two reviewers. Discrepancies were discussed and agreed.

\section{Assessment of study characteristics}

Data were extracted using a prepiloted form by one reviewer and checked by a second. The data included study characteristics (study design, selection criteria, setting and sample size), funding sources, ethical approval, population (age, gender, time since OVF), intervention and comparator characteristics (nature of intervention, duration of wear, concomitant interventions and adherence), outcomes, time points of follow-up and findings.

Study quality was assessed using the Cochrane Risk of Bias tool ${ }^{17}$ and was extracted by one reviewer and checked by a second. The data included random sequence generation, allocation concealment, blinding of participants and personnel, blinding of outcome assessment, incomplete outcome data, selective reporting and other sources of bias. Each item was rated as low risk, high risk or unclear risk of bias and reported for individual studies and across the studies.

\section{Data synthesis}

A narrative approach was used to synthesise the study findings due to methodological (study design, outcomes) and clinical (participant and intervention characteristics) heterogeneity. This approach enabled exploration of relationships within the data. ${ }^{18}$ Risk of bias was summarised for individual studies and across studies.

\section{RESULTS}

Our initial search resulted in 667 citations (figure 1) of which 84 were assessed against the selection criteria; the remainder did not meet the inclusion criteria. Nine studies were included in this review

\section{Characteristics of studies}

The studies included 468 participants of which 404 $(86 \%)$ were women. Where reported, participants had a mean (SD) age of 72.1 (7.9) years and had sustained an OVF between 3 days and 2 years previously. Six studies did not report time since fracture. The number of participants in each study varied from 13 to 108 (Mean=52). Studies took place in Europe ${ }^{19-22}$ Asia $^{23-26}$ and Australia. ${ }^{27}$ The majority of participants were ambulatory and community-dwelling. One study was undertaken with inpatients. ${ }^{25}$ Most studies took place in an outpatient setting with the setting being unclear in one study. ${ }^{26}$

Eight studies evaluated six different orthotic devices including rigid supports (TLSO, 3 point orthosis, plaster corset); semi-rigid supports (Spinomed and Spinomed Active); and flexible supports (soft brace) and one examined the effect of postural taping. Four studies evaluated two different types of orthotics. Controls included no brace, an alternative orthotic device (soft brace) or placebo (hypoallergenic tape). The devices were worn for between $15 \mathrm{~min}$ and $24 \mathrm{~h}$ a day (table 1). Adherence to wearing the orthotics was assessed by self-report in three studies ${ }^{21} 22 \quad 26$ although adherence was not defined. Additional data regarding individual study characteristics are reported in table 1 .

Two parallel-group randomised controlled trials, ${ }^{25} 26$ four randomised cross-over trials, ${ }^{21} \quad 22 \quad 24 \quad 27$ two 
Figure 1 Flow chart of study selection and inclusion.

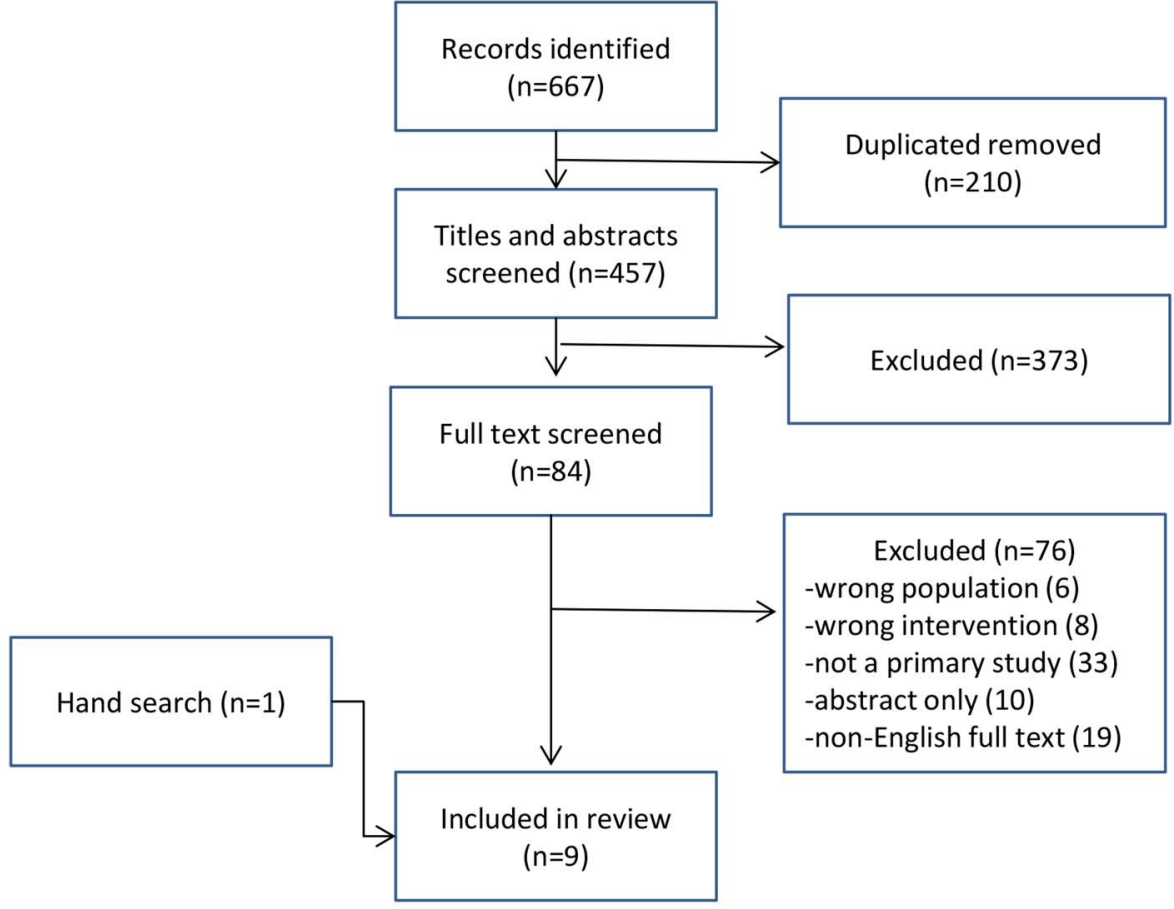

before-after (single arm) studies $^{19}{ }^{23}$ and a parallel group observational study ${ }^{20}$ were included. Three studies had three study arms. ${ }^{21} 2627$ No qualitative studies were identified. Of the four studies using crossover design, two did not report the findings from the first treatment period. ${ }^{24} 27$ The two studies undertaken by Pfeifer $e t a l^{1122}$ reported that almost all participants that received the intervention during the first period refused to stop using the device for the crossover. Therefore, to maintain clarity when comparing the different intervention groups in these two studies, only the findings from the first period were considered in this review.

\section{Study quality}

The results of the risk of bias assessment of study quality are presented in figure 2 and table 2. The reporting of studies was poor with no studies using ConSORT guidelines. ${ }^{28}$ Only one study reported the randomisation allocation processes. None had previously registered their studies or published a protocol. Four studies did not report ethical approvals ${ }^{20} 22-24$ and three did not report funding sources. ${ }^{20} 2327$ One study was termed a 'pilot' although the aims were stated to determine efficacy. ${ }^{25}$ Four studies undertook sample size calculations. ${ }^{21} 222627$ For the four studies using a cross-over design, additional quality assessments were made in relation to appropriate design, randomised treatment order, carry-over effect and unbiased data ${ }^{29}$ and these are recorded in table 2 under 'Other'.

\section{Effect of the intervention}

The effectiveness of orthotics and taping are reported in terms of impairments, activities and participation.
Follow-up varied from immediate, ${ }^{24} 27$ short term $(<1$ month), ${ }^{25}$ medium term (1-3 months) ${ }^{1926}$ and longer term (>3 months). ${ }^{20-23}$

\section{Impairments}

The majority of outcomes related to impairments such as pain, ${ }^{19-22} 2526$ postural stability, ${ }^{21} 2224$ back strength, ${ }^{19} 2122$ angle of kyphosis ${ }^{21-23} 2527$ and fracture union. $^{23}$

Pain: Two studies ${ }^{23}{ }^{26}$ targeted those with acute fractures using TLSO or soft brace and found no benefit, whereas those studies with those with longer term fractures reported mixed findings. Pfeifer et al reported the Spinomed device reduced pain measured using Milner's rating scale ( $1=$ low pain to $4=$ very severe pain). When compared with no brace they found an Absolute Difference $=-1.6 \quad(95 \%$ CIs -2.1 to -1.1$)$, with similar findings from an earlier study. ${ }^{21}{ }^{22}$ Valentin et al, ${ }^{19}$ however, did not find improvements in pain on a $0-10$ scale (with higher scores indicting worse pain) at 3 months when using Spinomed (median difference (range) $=-1 \quad(-4.7$ to 1.7$) ; \mathrm{p}=0.06)$. Li et $a l^{25}$ reported that Spinomed was no better than a soft brace after 3 weeks using a $0-10$ pain scale (mean pain (SD) 4.0 (2.0) vs $4.5(2.1))$.

Postural stability: A range of different methods were used to assess postural stability such as a force plate, ${ }^{27}$ computerised dynamic posturography ${ }^{24}$ and a sway metre. ${ }^{21}{ }^{22}$ Each assessment method reported multiple complex components of postural stability but there were no consistent findings within or across the studies. One cross-over study ${ }^{24}$ examined the immediate effect of the brace or no brace on balance performance but did not appear to consider potential carry-over effects nor 
Table 1 Characteristics of included studies

\begin{tabular}{|c|c|c|c|c|c|c|}
\hline & Study design & Study participants & $\begin{array}{l}\text { Sample } \\
\text { size }\end{array}$ & Intervention & Comparator & Follow-up \\
\hline Greig et $a^{R^{7}}$ & Crossover RCT & $\begin{array}{l}\text { Community dwelling women } \\
>50 \text {; OVF<2 years; post } \\
\text { menopause > } 5 \text { years; } \\
\text { confirmed osteoporosis }\end{array}$ & 15 & Therapeutic taping & $\begin{array}{l}\text { 1. Hypoallergenic } \\
\text { tape } \\
\text { 2. No tape }\end{array}$ & Immediate \\
\hline Kim et $a^{26}$ & Parallel group RCT & $\begin{array}{l}\text { Age }>50 \text {; acute back pain } \\
\text { from single OVF within } 3 \text { days } \\
\text { of minor trauma }\end{array}$ & 60 & $\begin{array}{l}\text { 1. Rigid TLSO for } \\
8 \text { weeks worn } \\
\text { continuously except } \\
\text { when lying down } \\
\text { 2. Soft brace for } \\
8 \text { weeks worn } \\
\text { continuously except } \\
\text { when lying down }\end{array}$ & No brace & 12 weeks \\
\hline Li et a ${ }^{55}$ & $\begin{array}{l}\text { Parallel group pilot } \\
\text { RCT }\end{array}$ & $\begin{array}{l}\text { Women }>55 \text {; OVF T1 to T5; } \\
\text { back pain due to OVF }\end{array}$ & 51 & $\begin{array}{l}\text { Spinomed brace } 3 \mathrm{~h} \text { a } \\
\text { day for } 2 \text { weeks+soft } \\
\text { brace remainder of day }\end{array}$ & $\begin{array}{l}\text { Soft brace worn all } \\
\text { day }\end{array}$ & 2 weeks \\
\hline Liaw et $a R^{4}$ & Crossover RCT & $\begin{array}{l}\text { Age 65-85; confirmed OVF; } \\
\text { severe osteoporosis }\end{array}$ & 47 & Rigid TLSO & No brace & Immediate \\
\hline Murata et $a^{P^{3}}$ & Before-after study & $\begin{array}{l}\text { Acute back pain }<1 \text { week; } \\
\text { confirmed OVF }\end{array}$ & 55 & $\begin{array}{l}\text { TLSO for at least } \\
2 \text { months ( } 24 \mathrm{~h} / \text { day } \\
\text { except for when } \\
\text { bathing) }\end{array}$ & - & 6 months \\
\hline Pfeifer et $a R^{2}$ & Crossover RCT & $\begin{array}{l}\text { Ambulatory community } \\
\text { dwelling women }>60 ; 1+\text { OVF; } \\
\text { kyphosis } \geq 60^{\circ}\end{array}$ & 62 & Spinomed for $2 \mathrm{~h}$ a day & No brace & 6 months \\
\hline Pfeifer et $a{ }^{21}$ & Crossover RCT & $\begin{array}{l}\text { Ambulatory community } \\
\text { dwelling women }>60 ; 1+\text { OVF; } \\
\text { kyphosis } \geq 60^{\circ}\end{array}$ & 108 & $\begin{array}{l}\text { 1. Spinomed for } 2 \mathrm{~h} \text { a } \\
\text { day } \\
\text { 2. Spinomed active for } \\
2 \text { hours a day }\end{array}$ & No brace & 6 months \\
\hline Talic et $a^{R o}$ & $\begin{array}{l}\text { Parallel group } \\
\text { observational study }\end{array}$ & OVF & 59 & $\begin{array}{l}\text { 1. Three-point orthosis } \\
\text { 2. Plaster corset }\end{array}$ & - & 4 months \\
\hline $\begin{array}{l}\text { Valentin } \\
\text { et al }\end{array}$ & Before-after study & $\begin{array}{l}\text { Women }>50 \text {; receiving } \\
\text { treatment for osteoporosis; } \\
\text { confirmed OVF; back pain } \\
>3 \text { months }\end{array}$ & 13 & $\begin{array}{l}\text { Spinomed worn for } \\
15 \mathrm{~min} / \text { day for } 2 \text { weeks; } \\
\text { then }<2 \mathrm{~h} \text { /day for } \\
2 \text { weeks; then } 2-4 \mathrm{~h} / \\
\text { day for } 8 \text { weeks }\end{array}$ & - & 3 months \\
\hline
\end{tabular}

report the findings from the first time period so it remains unclear as to the actual effect on postural stability of the orthosis.

Back strength: Three studies assessed the use of Spinomed on isometric back strength. ${ }^{19} 2122$ Pfeifer et $a l^{2122}$ reported a mean increase (SD) of 180 (152) Newtons when wearing the device and with an absolute difference of 182 (95\% CI 125.1 to 238.9) Newtons compared with wearing no brace.

Angle of kyphosis: Five studies reported angle of kyphosis using a range of techniques, including an inclinometer, ${ }^{27}$ radiographs ${ }^{23}{ }^{25}$ and three-dimensional photomorphometry. ${ }^{21} 22$ Postural taping was found to have immediate improvements in thoracic kyphosis when compared with placebo or no tape (Mean angle in degrees (SD) 55.3 (13.5); 57.2 (13.8); and, 58.2 (12.3) respectively; $\mathrm{p}=0.024) .{ }^{27} \mathrm{Li}$ et $a l^{25}$ only assessed this outcome on $10 / 51$ participants and it was unclear as to how they were selected. Two studies were unclear as to whether the findings represented an improvement or deterioration in kyphosis. ${ }^{23} 25$
Fracture union: Murata et $a l^{23}$ reported at 2 months $54.7 \%$ of participants had fracture 'settling'; with $88.7 \%$ settled at 6 months, however, the study did not have a control group and was, therefore, not possible to establish what benefit wearing the TLSO gave over 'normal' healing. The reporting of adverse events was poor with only one study explicitly identifying them as an outcome of interest. ${ }^{20}$ Talic et $a l^{20}$ found that plaster corsets resulted in an increased risk with four patients $(16 \%)$ developing pressure sores, with no adverse events related to using a three-point orthosis.

\section{Activities}

The impact of orthotics on activities was evaluated using the Oswestry Disability Index (ODI) ${ }^{26}$ the Functional Independence measure, Elderly Mobility Scale and Modified Functional Ambulation Category, ${ }^{25}$ limitations in everyday life $\mathrm{e}^{21} 22$ and walking ability component of the Japanese Orthopaedic Association Back Pain Evaluation Questionnaire (JOABPEQ) ${ }^{23}$ Pfeifer $e t a l^{21}{ }^{22}$ reported reduced disability associated with using 
Figure 2 Risk of bias graph indicating proportion of studies with each judgement.

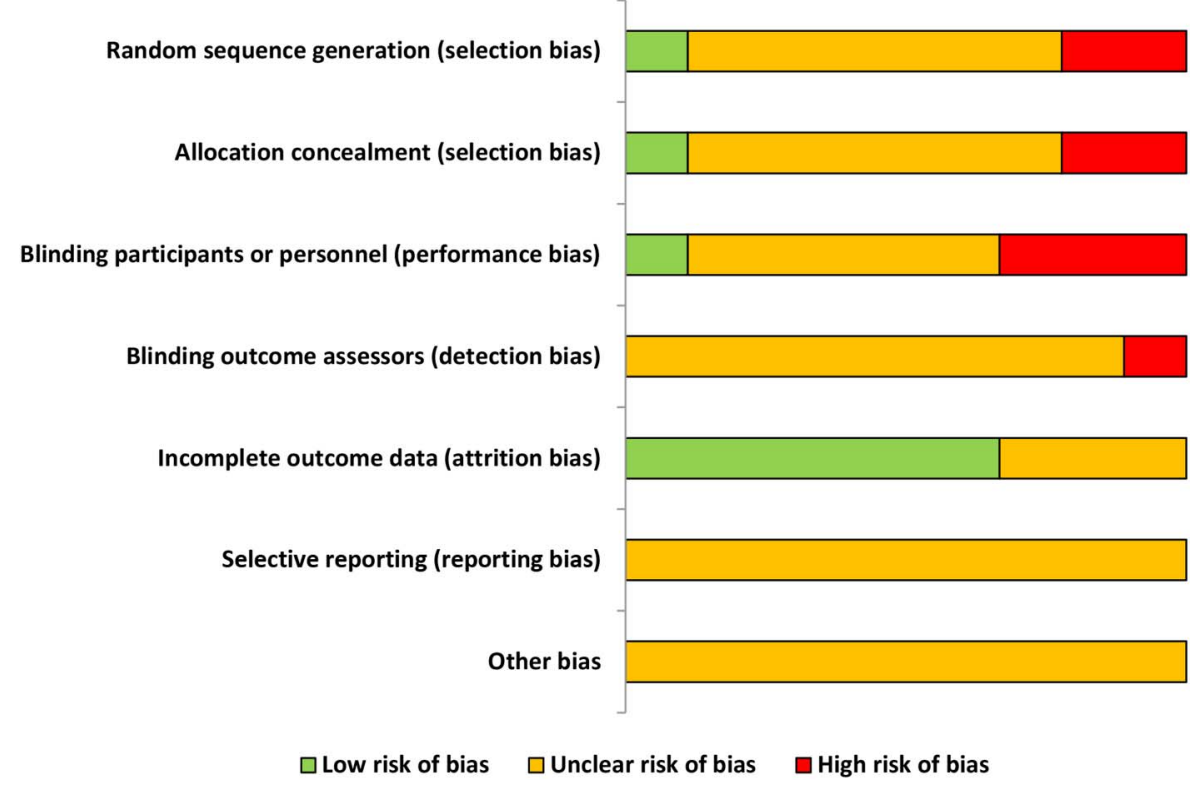

Spinomed when compared with no brace (absolute difference -2.3 (95\% CI -1.7 to -2.9$),{ }^{21}$ although it is unclear whether this is superior to a soft brace. ${ }^{25} \mathrm{Kim}$ $e t a l^{26}$ found no between group differences in ODI for those with an acute OVF when comparing TLSO (mean difference $-1.88 ; 95 \%$ CI -7.02 to 9.38 ) or soft bracing (mean difference $2.41 ;-7.86$ to 9.27 ) with no brace.

\section{Participation}

Participation, in relation to quality of life and well-being, was evaluated in four studies. ${ }^{19} 21 \quad 22 \quad 26$ The SF36 domains were not improved by using a TLSO or soft brace when compared with no brace (Mean Physical component score (PCS) 32, 35 and 30 respectively; $\mathrm{p}=0.716)^{26}$ or Spinomed (median within-group difference in Physical component score $=6.5$, range -9.2 to 13.3; $\mathrm{p}=0.07)^{19}$ whereas the Hobi well-being scale did improve after wearing Spinomed (absolute difference $=12.7$; $95 \%$ CI 9.7 to 15.7$).{ }^{21}$ There was no indication that either outcome was moderated by population characteristics.

\section{DISCUSSION}

This review aimed to establish the effectiveness of orthotics or taping in the management of OVF. We found that the nine included studies were of limited quality and reported varied populations, interventions and outcomes. For example, no studies reported whether outcome assessors were blinded to allocation, thus the potential for detection bias is unknown. A previous review of the non-surgical management of OVF included three RCTs evaluating bracing ${ }^{30}$ reported medium term pain relief and reduced disability; however, studies were considered to be of low/very low quality. We found little consideration of any potential adverse events associated with bracing or taping with this population. It has been suggested that adherence to wearing orthotics is poor ${ }^{31}$ but despite several included studies stating adherence data was collected it was never defined and rarely reported. In addition, there is a complete absence of qualitative research involving this population and their experiences of wearing these devices which would be an essential component of any future development and evaluation. This said, in two cross-over studies, ${ }^{21}{ }^{22}$ the fact that the participants refused to stop wearing the orthotics at the point of crossover would suggest positive experiences.

We found no evidence to counter the recommendations of the American Academy of Orthopaedic Surgeons ${ }^{13}$ that indicated there was inconclusive evidence to support the using of bracing in the management of OVF, and that the quality of studies in this clinical area remains limited. Our findings also concur with a recent systematic review of taping that reported it was no better than placebo or no taping in terms of pain or disability for people with back pain. ${ }^{32}$

In terms of strengths, our study used contemporary methods for undertaking a systematic review and registered the protocol prospectively on PROSPERO. We searched an extensive range of databases, including grey literature. One limitation was the exclusion of non-English full-text papers, however, we are confident from our extensive search (that was not restricted to English) and screening process that any potential papers would have failed to meet other selection criteria and therefore would not have been included in the review. It could also be suggested that including non-randomised and non-controlled studies is a limitation. However, the purpose of our review was to establish the current evidence base, and not establish effectiveness, and therefore we considered it important to include all study designs. In addition, the use of the risk of bias tool enabled a judgement on the overall quality of the 


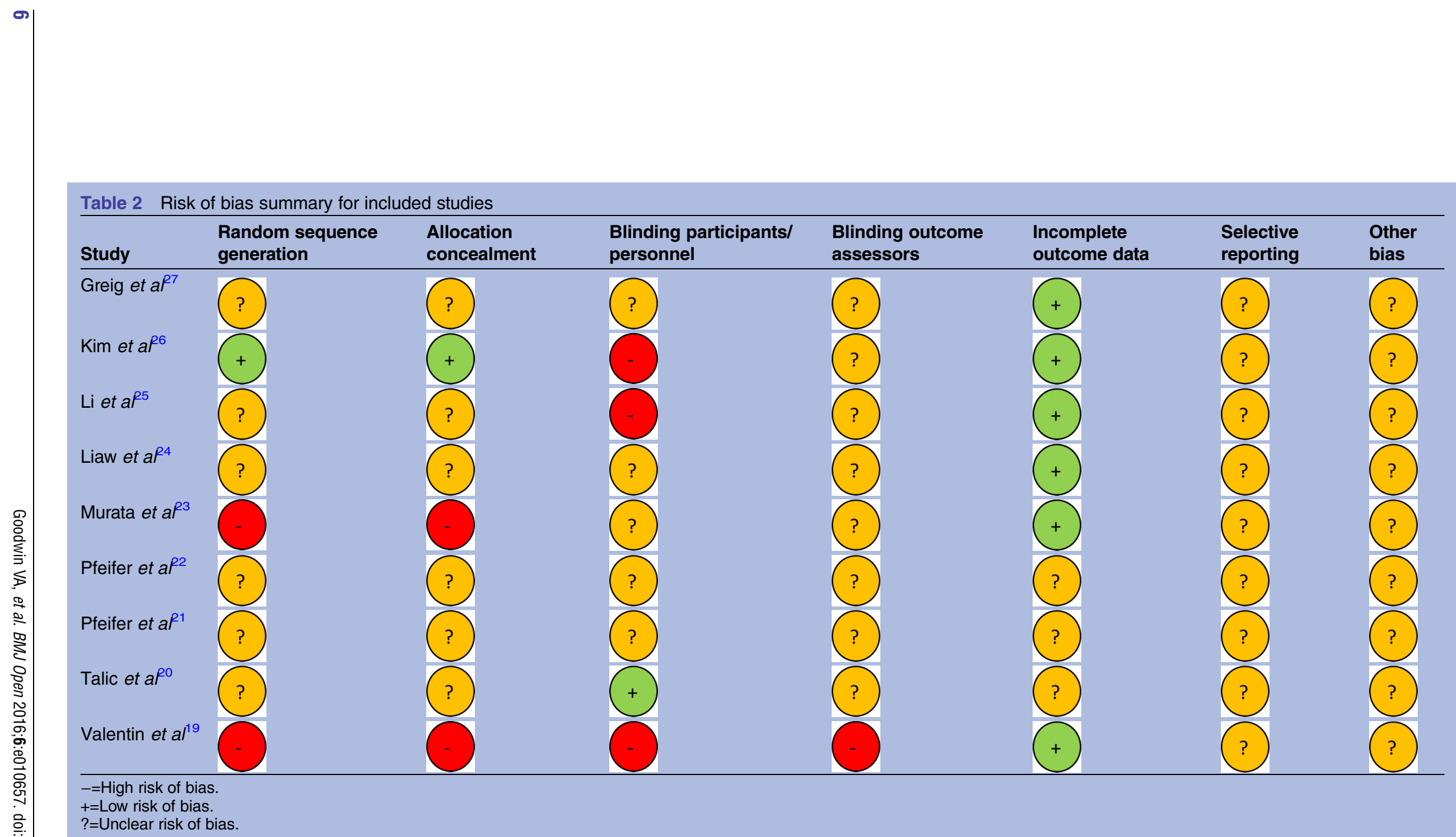

Table 2 Risk of bias summary for included studies

Study
Greig $e t a P^{7}$


included studies, and we found that even the included randomised controlled trials were not without limitations.

\section{CONCLUSIONS}

There is inconclusive evidence that TLSO or soft braces reduce pain or disability. Although the use of the Spinomed appears to have some benefit in terms of increasing back strength and reducing disability, it does not necessarily offer better outcomes when compared with other devices, such as soft braces. The quality of studies examining the effectiveness of orthotics or taping for the management of OVF is generally limited and therefore we would err on the side of caution when considering their use in clinical practice. Overall, there is limited evidence for the use of orthotics or taping either in the acute or long-term management of those with OVF. Further studies using high-quality methods and reporting are required to determine whether taping or orthotics are effective and cost-effectiveness.

Contributions VAG, AJH and $A B$ developed the study protocol. $A H$ and $A B$ performed the searches. VG and $A H$ undertook all screening and all authors were involved in data extraction. VG undertook the synthesis. All authors contributed to the final manuscript.

Funding This review was supported by a research grant from AGILE (Chartered Physiotherapists working with Older People) and also by the National Institute for Health Research (NIHR) Collaboration for Leadership in Applied Health Research and Care South West Peninsula. The views expressed are those of the authors and not necessarily those of the NHS, the NIHR or the Department of Health.

Competing interests None declared.

Provenance and peer review Not commissioned; externally peer reviewed.

Data sharing statement No additional data are available.

Open Access This is an Open Access article distributed in accordance with the terms of the Creative Commons Attribution (CC BY 4.0) license, which permits others to distribute, remix, adapt and build upon this work, for commercial use, provided the original work is properly cited. See: http:// creativecommons.org/licenses/by/4.0/

\section{REFERENCES}

1. Gerdhem P. Osteoporosis and fragility fractures: vertebral fractures. Best Pract Res Clin Rheumatol 2013;27:743-55.

2. Felsenberg D, Silman AJ, Lunt M, et al. Incidence of vertebral fracture in Europe: results from the European Prospective Osteoporosis Study (EPOS). J Bone Miner Res 2002;17:716-24.

3. Ensrud KE, Schousboe JT. Clinical practice. Vertebral fractures. N Engl J Med 2011;364:1634-42.

4. Francis RM, Baillie SP, Chuck AJ, et al. Acute and long-term management of patients with vertebral fractures. QJM 2004;97:63-74.

5. Gauthier A, Kanis JA, Jiang Y, et al. Epidemiological burden of postmenopausal osteoporosis in the UK from 2010 to 2021: estimations from a disease model. Arch Osteoporos 2011;6:179-88.

6. Wong CC, McGirt MJ. Vertebral compression fractures: a review of current management and multimodal therapy. J Multidiscip Healthc 2013;6:205-14.

7. Triantafyllopoulos IK, Lambropoulou-Adamidou K, Nacopoulos CC, et al. EMAS position statement: the management of postmenopausal women with vertebral osteoporotic fracture. Maturitas 2014;78:131-7

8. Waterloo S, Søgaard AJ, Ahmed LA, et al. Vertebral fractures and self-perceived health in elderly women and men in a population-based cross-sectional study: the Tromsø Study 2007-08. BMC Geriatr 2013;13:102.

9. Briggs AM, Greig AM, Wark JD. The vertebral fracture cascade in osteoporosis: a review of aetiopathogenesis. Osteoporos Int 2007; 18:575-84.

10. van der Jagt-Willems HC, Vis M, Tulner CR, et al. Mortality and incident vertebral fractures after 3 years of follow-up among geriatric patients. Osteoporos Int 2013;24:1713-19.

11. Dolan P, Torgerson DJ. The cost of treating osteoporotic fractures in the United Kingdom female population. Osteoporos Int 1998;8:611-17.

12. Cooper C. Epidemiology and public health impact of osteoporosis. In: Reid D, ed. Bailliere's clinical rheumatology: osteoporosis. Bailliere Tindall, 1993:459-77.

13. Esses SI, McGuire R, Jenkins J, et al. American Academy of Orthopaedic Surgeons clinical practice guideline on: the treatment of osteoporotic spinal compression fractures. J Bone Joint Surg Am 2011;93:1934-6.

14. Scottish Intercollegiate Guidelines Network. Management of osteoporosis and the prevention of fragility fractures: a national clinical guideline. Edinburgh: Health Improvement Scotland, 2015.

15. Babb A, Carlson WO. Vertebral compression fractures: treatment and evaluation. S D Med 2006;59:343-5, 47.

16. Giele BM, Wiertsema $\mathrm{SH}$, Beelen A, et al. No evidence for the effectiveness of bracing in patients with thoracolumbar fractures. Acta Orthop 2009;80:226-32.

17. Higgins JPT, Green S. Cochrane handbook for systematic reviews of interventions Version 5.1.0. Chichester, UK: The Cochrane Collaboration, 2011.

18. Pope C, Mays N, Popay J. Synthesizing qualitative and quantitative health evidence. Maidenhead, UK: Open University Press, 2007.

19. Valentin GH, Pedersen LN, Maribo T. Wearing an active spinal orthosis improves back extensor strength in women with osteoporotic vertebral fractures. Prosthet Orthot Int 2014:38:232-8.

20. Talic A, Kapetanovic J, Dizdar A. Effects of conservative treatment for osteoporotic thoracolumbal spine fractures. Mater Sociomed 2012;24:16-20.

21. Pfeifer M, Kohlwey L, Begerow B, et al. Effects of two newly developed spinal orthoses on trunk muscle strength, posture, and quality-of-life in women with postmenopausal osteoporosis: a randomized trial. Am J Phys Med Rehabil 2011;90:805-15.

22. Pfeifer M, Begerow B, Minne HW. Effects of a new spinal orthosis on posture, trunk strength, and quality of life in women with postmenopausal osteoporosis: a randomized trial. Am J Phys Med Rehabil 2004:83:177-86.

23. Murata K, Watanabe G, Kawaguchi S, et al. Union rates and prognostic variables of osteoporotic vertebral fractures treated with a rigid external support. J Neurosurg Spine 2012;17:469-75.

24. Liaw MY, Chen CL, Chen JF, et al. Effects of Knight-Taylor brace on balance performance in osteoporotic patients with vertebral compression fracture. J Back Musculoskelet Rehabil 2009;22: 75-81.

25. Li M, Law SW, Cheng J, et al. A comparison study on the efficacy of SpinoMed $\circledast$ and soft lumbar orthosis for osteoporotic vertebral fracture. Prosthet Orthot Int 2015;39:270-6.

26. Kim HJ, Yi JM, Cho HG, et al. Comparative study of the treatment outcomes of osteoporotic compression fractures without neurologic injury using a rigid brace, a soft brace, and no brace: a prospective randomized controlled non-inferiority trial. J Bone Joint Surg Am 2014;96:1959-66.

27. Greig AM, Bennell KL, Briggs AM, et al. Postural taping decreases thoracic kyphosis but does not influence trunk muscle electromyographic activity or balance in women with osteoporosis. Man Ther 2008;13:249-57.

28. Moher D, Schulz KF, Altman DG. The CONSORT statement: revised recommendations for improving the quality of reports of parallel-group randomised trials. Lancet 2001;357: 1191-4.

29. Ding H, Hu GL, Zheng XY, et al. The method quality of cross-over studies involved in Cochrane Systematic Reviews. PLoS One 2015;10:e0120519.

30. Rzewuska M, Ferreira M, McLachlan AJ, et al. The efficacy of conservative treatment of osteoporotic compression fractures on acute pain relief: a systematic review with meta-analysis. Eur Spine J 2015;24:702-14.

31. Lin JT, Lane JM. Nonmedical management of osteoporosis. Curr Opin Rheumatol 2002;14:441-6.

32. Vanti C, Bertozzi L, Gardenghi I, et al. Effect of taping on spinal pain and disability: systematic review and meta-analysis of randomized trials. Phys Ther 2015;95:493-506. 